\title{
Varying Estimates of Sepsis Mortality Using Death Certificates and Administrative Codes — United States, 1999-2014
}

\author{
Lauren Epstein, $\mathrm{MD}^{1}$; Ray Dantes, $\mathrm{MD}^{1}$; Shelley Magill, MD, $\mathrm{PhD}^{1}$; Anthony Fiore, $\mathrm{MD}^{1}$
}

Sepsis is a clinical syndrome caused by a dysregulated host response to infection (1). Because there is no confirmatory diagnostic test, the diagnosis of sepsis is based on evidence of infection and clinical judgement. Both death certificates and health services utilization data (administrative claims) have been used to assess sepsis incidence and mortality, but estimates vary depending on the surveillance definition and data source. To highlight the challenges and variability associated with estimating sepsis mortality, CDC compared national estimates of sepsis-related mortality based on death certificates using the CDC WONDER database with published sepsis mortality estimates generated using administrative claims data from hospital discharges reported in the Nationwide Inpatient Sample, Healthcare Cost and Utilization Project, Agency for Healthcare Research and Quality (2). During 2004-2009, using data rounded to thousands, the annual range of published sepsisrelated mortality estimates based on administrative claims data was $15 \%$ to $140 \%$ higher $($ range $=168,000-381,000)$ than annual estimates generated using death certificate data (multiple causes) (range $=146,000-159,000)$. Differences in sepsis-related mortality reported using death certificates and administrative claims data might be explained by limitations inherent in each data source. These findings underscore the need for a reliable sepsis surveillance definition based on objective clinical data to more accurately track national sepsis trends and enable objective assessment of the impact of efforts to increase sepsis awareness and prevention.

Death certificate data were obtained from multiple cause-ofdeath records of the National Vital Statistics System, using CDC's WONDER database (http://wonder.cdc.gov/mcd.html). Multiple cause-of-death records include the immediate cause of death (i.e., the final disease or condition resulting in death), up to 20 contributing causes, a single underlying cause of death (i.e., the disease or injury that initiated the events resulting in death), and significant conditions that were present at the time of death but were not a direct link in the chain of events leading to death. All information on death certificates is documented by the certifier (e.g., a physician, medical examiner, or coroner) and subsequently coded by the National Center for Health Statistics in accordance with guidelines specified by the World Health Organization (3). National trends in sepsis-related mortality have been previously estimated from administrative claims data obtained from the Nationwide Inpatient Sample, the largest all-payer, publicly-available inpatient database in the United States, using various combinations of the International Classification of Diseases 9th Revision Clinical Modification (ICD-9-CM) administrative codes for primary or secondary infection and organ dysfunction to identify severe sepsis.

In a published report (2), investigators generated a range of sepsis mortality estimates using four previously established approaches to identifying adult patients (aged $\geq 18$ years) with sepsis using administrative claims data. Two of these approaches $(4,5)$ defined sepsis using explicit, sepsis-specific ICD-9-CM codes in addition to various codes for infection and organ dysfunction, whereas the other two approaches $(6,7)$ defined sepsis on the basis of combinations of infection criteria and organ dysfunction as implicit markers for severe sepsis.

Multiple cause-of-death mortality files maintained in the CDC WONDER database were reviewed to analyze deaths (for all ages) from sepsis reported on death certificates during 1999-2014, defined as deaths with diagnoses corresponding to International Classification of Diseases 10th Revision (ICD-10) diagnosis codes A40 (streptococcal septicemia) and A41 (other septicemia) listed on the death certificate. The annual sepsis mortality estimates based on death certificates from the WONDER database were then compared with the previously published annual estimates generated based on the ICD-9-CM administrative codes data from the Nationwide Inpatient Sample.

Based on multiple cause-of-death data during 1999-2014, a total of 2,470,666 decedents (6\% of all deaths) had sepsis listed among the causes of death (sepsis-related deaths); for $22 \%$ of these decedents, sepsis was listed as the underlying cause of death. During this period, the annual number of all reported sepsis-related deaths increased 31\%, from 139,086 in 1999 to 182,242 in 2014 (Figure 1). Approximately 15\% of all sepsis-related deaths during this period occurred in nonacute care settings (e.g., at home, long-term care facilities, hospice, and unknown setting). Among the 2,472,911 A40 and A41 codes listed for the 2,470,666 decedents, the most common were unspecified septicemia (A41.9, 94\%), septicemia caused by other gram-negative organisms (A41.5, 2\%), and septicemia caused by Staphylococcus aureus (A41.0, 2\%). Among decedents, approximately $49 \%$ were aged $65-84$ years, $26 \%$ were aged $\geq 85$ years, and $25 \%$ were aged $25-64$ years ( $4 \%$ aged 
FIGURE 1. Comparison of sepsis-related mortality estimates based on death certificates* (1999-2014) with four estimates from administrative claims data ${ }^{\dagger}$ (2004-2009) - United States

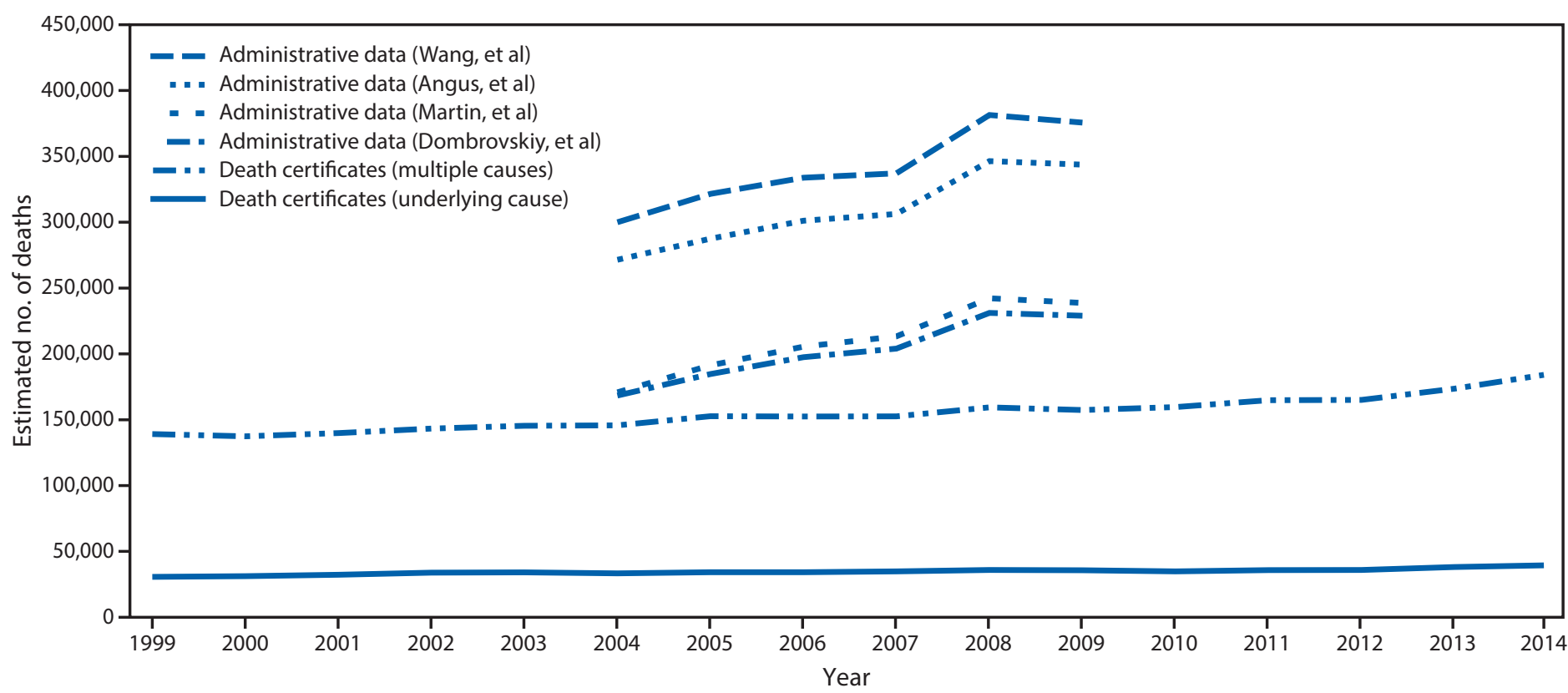

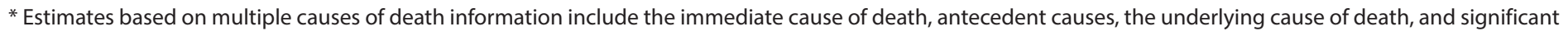

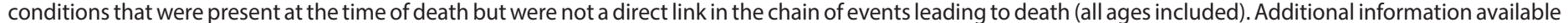

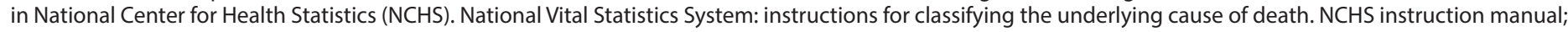

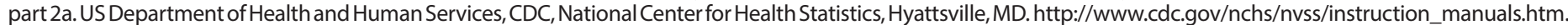

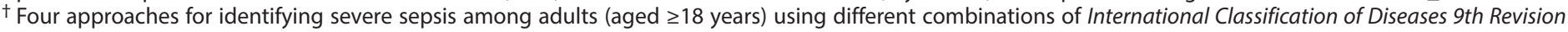

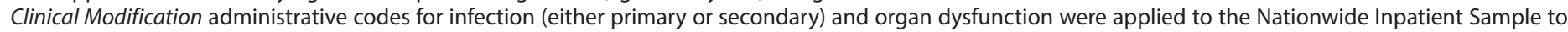
generate a range of sepsis-related mortality estimates for the period 2004-2009. Additional information available in the following:

Gaieski DF, Edwards M, Kallan MJ, Carr BG. Benchmarking the incidence and mortality of severe sepsis in the United States. Crit Care Med 2013;41:1167-74.

Wang HE, Shapiro NI, Angus DC, Yealy DM. National estimates of severe sepsis in United States emergency departments. Crit Care Med 2007;35:1928-36.

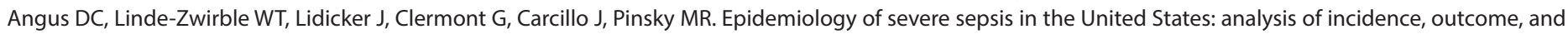
associated costs of care. Crit Care Med 2001;29:1303-10.

Martin GS, Mannino DM, Eaton S, Moss M. The epidemiology of sepsis in the United States from 1979 through 2000. N Engl J Med 2003;348:1546-54.

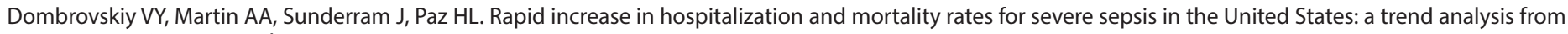
1993 to 2003. Crit Care Med 2007;35:1244-50.

25-44 years and 21\% aged 45-64 years). Approximately 1\% of decedents were aged $<25$ years. (Figure 2 ).

During 2004-2009, using data rounded to thousands, the annual range of published sepsis-related mortality estimates based on administrative codes (range $=168,000-381,000$ ) was $15 \%$ to $140 \%$ higher than annual estimates generated using death certificates (multiple causes) for those years $($ range $=146,000-159,000)($ Figure 1$)$.

\section{Discussion}

Death records and administrative claims data are important sources of public health information. However, they provide different estimates of sepsis mortality, and recognizing the limitations of both methods for generating sepsis-related mortality estimates is important.

Death certificate certifiers are required to indicate as the underlying cause of the death a specific disease or injury that initiated the chain of events leading directly to death (3).
Because sepsis is the final common pathway for many different severe infections, certifiers might be more likely to consider sepsis an immediate, rather than the underlying, cause of death. This practice might result in an underestimation of the importance of sepsis as a cause of death and lower estimates of sepsis-related mortality that are based on the underlying causes of death only (8). Furthermore, because of misunderstandings regarding the process of completing death certificates, certifiers might omit sepsis entirely as a cause of death on the death certificate. For example, the New York City medical examiner's policy of rejecting New York City death certificates with sepsis listed alone as the single underlying cause of death (without other contributing causes) has been misinterpreted by some clinicians to mean that the term "sepsis" was not permitted to be included anywhere on the death certificate, thus affecting estimates of sepsis-related mortality (8). In addition, it is important to note that death certificates are often completed by physicians with varying levels of training in completing 
FIGURE 2. Percentage of sepsis-related deaths $(N=2,470,666)$ based on death certificate data, by age groups* - United States, 1999-2014

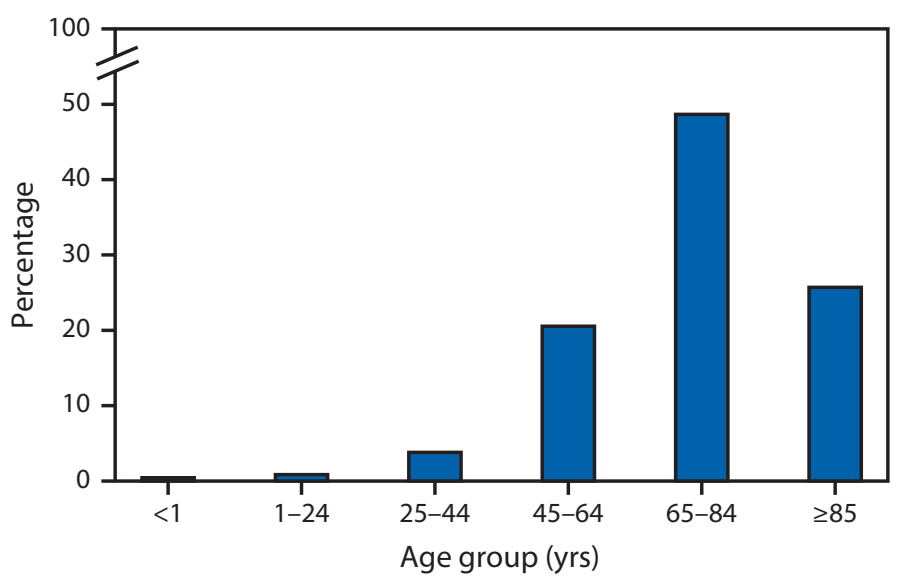

* Age was unknown for 90 decedents.

death certificates; data from a survey of resident physicians in New York City showed that only $40 \%$ reported receiving any training by their residency program regarding completion of death certificates (9). Finally, sepsis can be confused with other acute medical problems, such as myocardial infarction, that frequently cause mortality in the community setting.

Estimates based on administrative claims data also have limitations. Previous analyses of administrative claims data have shown that estimates of sepsis incidence have increased over time, whereas diseases and conditions that can cause sepsis (e.g., pneumonia or urinary tract infections) have decreased or remained stable (10). Increased reimbursement for sepsis (compared with other infectious disease diagnoses) encourages hospitals to be more careful about coding for sepsis. Improved recognition and awareness of sepsis have also likely contributed to increased documentation of sepsis over time (10). Finally, administrative data only capture sepsis-related deaths that occur within health care facilities. However, approximately $15 \%$ of sepsis-related deaths recorded in death certificate data occurred outside of health care facilities, suggesting sepsis mortality estimates based on administrative data might not include these deaths.

Sepsis is a complex clinical syndrome, representing a response to infection that can arise from many underlying causes. Differences in sepsis-related mortality estimates derived from death certificates and administrative claims data might be explained by limitations inherent in each data source. Current efforts focused on evaluating linkages of administrative claims data derived from inpatient medical records with death certificates will improve understanding of how mortality estimates from death certificates and administrative claims data should be

\section{Summary \\ What is already known about this topic?}

Sepsis is a clinical syndrome caused by response to infection. Because there is no confirmatory diagnostic test, the diagnosis of sepsis is based on clinical judgement of suspected infection. Data from both death certificate and administrative claims data have been used to assess sepsis incidence and mortality, but estimates vary depending on the surveillance definition and data source.

What is added by this report?

To highlight the challenges and variability associated with estimating sepsis mortality, CDC compared national estimates of sepsis-related mortality based on death certificates with previously published sepsis mortality estimates generated using administrative claims data. Using death certificate data for the period 1999-2014, CDC found that a total of 2,470,666 decedents ( $6 \%$ of all deaths) had sepsis listed among the causes of death (sepsis-related deaths); for $22 \%$ of these decedents, sepsis was listed as the underlying cause of death. For the period 2004-2009, in a previously published report, investigators analyzed administrative claims data using four approaches for identifying adult patients (aged $\geq 18$ years) with sepsis. In data rounded to thousands, the annual range of published sepsis-related mortality estimates based on administrative claims data was $15 \%$ to $140 \%$ higher (range $=168,000-381,000$ ) than annual estimates generated using death certificate data (multiple causes) (range $=146,000-159,000$ ).

What are the implications for public health practice?

Sepsis is a complex clinical syndrome, representing a response to infection that can arise from many different underlying causes. A reliable sepsis surveillance definition based on objective clinical data is needed to more accurately track national sepsis trends and enable ongoing assessment of the impact of efforts to increase sepsis awareness and prevention.

interpreted. Strategies to prevent infections that lead to sepsis include vaccination for pathogens like pneumococcus, influenza, and Neisseria meningitidis, smoking cessation programs to reduce the risk for community-acquired pneumonia, and facility-level interventions to reduce risk for health care-associated infections. A reliable sepsis surveillance definition based on objective clinical data is needed to more accurately track national sepsis trends and enable ongoing assessment of the impact of efforts to increase sepsis awareness and prevention.

\section{Acknowledgment}

David F. Gaieski, MD, Department of Emergency Medicine, Thomas Jefferson University Hospital, Philadelphia, Pennsylvania.

\footnotetext{
${ }^{1}$ Division of Healthcare Quality Promotion, National Center for Emerging and Zoonotic Infectious Diseases, CDC.

Corresponding author: Lauren Epstein, lepstein@cdc.gov, 404-639-8162.
} 


\section{References}

1. Singer M, Deutschman CS, Seymour CW, et al. The third international consensus definitions for sepsis and septic shock (sepsis-3). JAMA 2016;315:801-10. http://dx.doi.org/10.1001/jama.2016.0287

2. Gaieski DF, Edwards JM, Kallan MJ, Carr BG. Benchmarking the incidence and mortality of severe sepsis in the United States. Crit Care Med 2013;41:1167-74. http://dx.doi.org/10.1097/CCM.0b013e31827c09f8

3. National Center for Health Statistics (NCHS). National Vital Statistics System: instructions for classifying the underlying cause of death. NCHS instruction manual; part 2a. Hyattsville, MD: US Department of Health and Human Services, CDC, National Center for Health Statistics; 2016. http://www.cdc.gov/nchs/nvss/instruction_manuals.htm

4. Wang HE, Shapiro NI, Angus DC, Yealy DM. National estimates of severe sepsis in United States emergency departments. Crit Care Med 2007;35:192836. http://dx.doi.org/10.1097/01.CCM.0000277043.85378.C1

5. Dombrovskiy VY, Martin AA, Sunderram J, Paz HL. Rapid increase in hospitalization and mortality rates for severe sepsis in the United States: a trend analysis from 1993 to 2003. Crit Care Med 2007;35:1244-50. http://dx.doi.org/10.1097/01.CCM.0000261890.41311.E9
6. Martin GS, Mannino DM, Eaton S, Moss M. The epidemiology of sepsis in the United States from 1979 through 2000. N Engl J Med 2003;348:1546-54. http://dx.doi.org/10.1056/NEJMoa022139

7. Angus DC, Linde-Zwirble WT, Lidicker J, Clermont G, Carcillo J, Pinsky MR. Epidemiology of severe sepsis in the United States: analysis of incidence, outcome, and associated costs of care. Crit Care Med 2001;29:1303-10. http://dx.doi.org/10.1097/00003246-200107000-00002

8. Ong P, Gambatese M, Begier E, Zimmerman R, Soto A, Madsen A. Effect of cause-of-death training on agreement between hospital discharge diagnoses and cause of death reported, inpatient hospital deaths, New York City, 2008-2010. Prev Chronic Dis 2015;12:140299. http://dx.doi.org/10.5888/pcd12.140299

9. Wexelman BA, Eden E, Rose KM. Survey of New York City resident physicians on cause-of-death reporting, 2010. Prev Chronic Dis 2013;10:120288. http://dx.doi.org/10.5888/pcd10.120288

10. Rhee C, Gohil S, Klompas M. Regulatory mandates for sepsis carereasons for caution. $N$ Engl J Med 2014;370:1673-6. http://dx.doi. org/10.1056/NEJMp1400276 